\title{
Fetal Developmental Deviations Reflected in a Functional Autonomic Brain Age Score
}

\author{
Dirk Hoyer ${ }^{1}$, Alexander Schmidt ${ }^{1}$, Uwe Schneider ${ }^{2}$, Kathleen Gustafson ${ }^{3}$ \\ ${ }^{1}$ Biomagnetic Center, Hans Berger Department of Neurology, Jena University Hospital, Germany \\ ${ }^{2}$ Department of Obstetrics, Jena University Hospital, Germany \\ ${ }^{3}$ Hoglund Brain Imaging Center, Department of Neurology, University of Kansas Medical Center, \\ USA
}

\begin{abstract}
The functional maturation of the fetal autonomic control can be evaluated by means of the fetal Autonomic Brain Age Score (fABAS) that constitutes a non-invasively recordable index of the neural integration of the developing organism. We wanted to know how maternal docosahexaenoic acid (DHA) supplementation that was previously shown to increase traditional fetal and neonatal heart rate variability (HRV) as well as autonomic clusters of Neonatal Behavioral Assessment Scale also influences $f A B A S$.

Women were randomized to groups of daily intake of either $600 \mathrm{mg}$ of DHA or placebo during the last two trimesters of pregnancy. $f A B A S$ and the included $H R V$ indices were calculated from fetal $R R$ interval series obtained from magnetocardiographic recordings.

fABAS was increased in the DHA group at 32 and 36 weeks of gestation (WGA), but by tendency at 24 WGA. Separately considering the factors of fABAS, pNN5 was increased in the DHA group from 32 WGA onwards but ActAmp at 36 WGA only.

The combined interpretation of $f A B A S$ and its coefficients may provide advanced understanding of the complex neural interactions during fetal development with implementations on age depending nutritional programming effects.
\end{abstract}

\section{Introduction}

According to the "developmental origins of health and disease" hypothesis (Barker [1]) developmental disturbances during the fetal period can have irreversible implications for health problems in the later life. Therefore, early identification of fetal developmental problems and effective therapeutic interventions becomes critically important. To this end, a sensitive biomarker of fetal autonomic development is needed.

The Fetal Autonomic Brain Age Score (fABAS) is based on a system biology approach that applies universal principles of evolution and self-organization to the ontogenetic functional development of the fetus, mainly mediated by the autonomic nervous system (ANS). It can be understood as a proxy for the neural integration of the developing organism. fABAS is calculated from the fetal cardiac autonomic outflow using fetal heart rate variability (fHRV) indices. The coefficients of fABAS resulted from a multivariate linear regression procedure using stepwise parameter selection with respect to fHRV of normal developing fetuses between 20 and 36 weeks of gestation (WGA) [2].

Several fetal developmental disturbances were reported as changing traditional fetal HRV indices (e.g. [3, 4]). But effects on the fetal functional autonomic brain age were mainly not yet investigated. First results showed reduced fABAS values in intrauterine growth restricted fetuses [2, 5]. A comprehensive evaluation of the diagnostic value of fABAS with respect to different developmental influences as well as its comparison to traditional fHRV indices is pending. In the present work we want to determine how maternal docosahexaenoic acid (DHA) supplementation influences fetal cardiac autonomic outflow assessed by fABAS.

DHA is a long-chain polyunsaturated fatty acid essential for fetal brain and nervous system development. Besides several other effects on cellular mechanisms it stimulates neural growth and cellular communication. Recently, it was shown that maternal DHA supplementation increases traditional fHRV as well as autonomic and motor clusters of the Neonatal Behavioral Assessment Scale (NBAS) [6]. In particular, the NBAS autonomic cluster reflects the newborn's physiological reactivity and ability to adjust to the environment. Mature autonomic function includes developed flexibility and 
integrity of the ANS. The effects of DHA on development may be mediated by the ANS [7]. Consequently, we expect enhanced fABAS values in the DHA supplemented group.

By means of a reanalysis of the previous study data set [6] we want to address the following methodological questions. (1.) What is the effect of maternal DHA supplementation on fABAS compared to placebo? (2.) Is the DHA effect, expressed by the contribution of the fABAS coefficients, symmetrical with respect to the physiological aging?

\section{Methods}

fABAS [2] estimates functional brain age by means of a linear model based on evolution-related fHRV indices:

ActAmp: The typically appearing increase of fluctuation range was estimated in a robust way by the 20-95 inter quantile distance of detrended NN intervals, denoted ActAmp. All kind of autonomic, such as vagal and sympathetic, rhythms as well as humoral influences are considered with respect to their integrated processing.

pNN5: The increasing amplitude of fast fluctuations emphasizes the role of vagal rhythms that are intrinsically linked to sympathetic activity in both, antagonistic and synergetic way, are assessed by the percentage of $\mathrm{NN}$ intervals larger than $5 \mathrm{~ms}$.

Skewness: The typical pattern formation is reflected in the skewness, denoted by skew. During the fetal maturation there is a shift from predominant heart rate decelerations at gestational ages before 32 WGA to predominant and increasing heart rate accelerations later on.

VLF/LF: The interpretation of the ratio very low to low frequency band power, denoted by VLF/LF, is diverse. David et al. [8] proposed sympatho-vagal balance. In the context of developmental principles increasing $\mathrm{VLF} / \mathrm{LF}$ can be explained by increasing correlations over time and forming longer lasting patterns or by accidental heart rate baseline fluctuations.

gMSE3: The typical complexity increase was previously found at coarse graining time scales around 35 heart beat intervals. At these scales the heart rate fluctuations result from the complex interplay of vagal and sympathetic processes. We analyzed the complexity at scale 3 by generalized mutual information which is a robust estimator due to its rank based statistic.

The calculation of the fHRV indices and fABAS was done based on moving $7 \mathrm{~min}$ windows and under exclusion of heart rate deceleration. For details see Schmidt et al. in this issue. fABAS was fitted to $n=482$ normal fetuses of the Jena data base (Study Group 'Prenatal Monitoring of Fetal Maturation', Department of
Obstetrics, Division of Prenatal Diagnostics and Fetal Physiology; Biomagnetic Center, Hans Berger Department of Neurology, both Jena University Hospital).

As previously reported [6] 67 women were randomized to either placebo (corn/soy oil) or a daily $600 \mathrm{mg}$ of DHA supplementation during the last two trimesters of pregnancy (NCT01007110) at the Hoglund Brain Imaging Center, Department of Neurology, University of Kansas Medical Center, Kansas City. At 24 WGA we considered $n=19$ women in the placebo group vs. $n=22$ in the DHA group, at 32 WGA $n=25$ vs. $n=23$, and at 36 WGA $n=24$ vs. $n=22$, respectively.

fABAS was calculated from RR interval series from two 18 min blocks of magnetocardiographic recordings and fetal activity states, namely quite sleep 1F, active sleep $2 \mathrm{~F}$, and active wakefulness $4 \mathrm{~F}$ were classified by consensus decision of 3 experts. The RR interval series were checked for artifacts and outliers that were deleted. Their frequency did not exceed $2 \%$ of the individual data sets.

According to recommendations [9] only sections in active sleep $(2 \mathrm{~F})$ were considered in both data sets.

The results of the DHA group are presented by the calculated fABAS values and their z-scored values normalized to the placebo group in a modified forest plot like fashion. The dashed lines indicate mean and standard deviation of the placebo group. The groups were compared by Mann-Whitney-U-test.

\section{Results}

Due to the lack of analyzable sections on active sleep $2 \mathrm{~F}$ in 13 cases, we analyzed at $24 \mathrm{WGA} n=16$ women in the placebo group vs. $\mathrm{n}=19$ in the DHA group, at 32 WGA $n=24$ vs. $n=23$, and at 36 WGA $n=19$ vs. $n=21$, respectively.

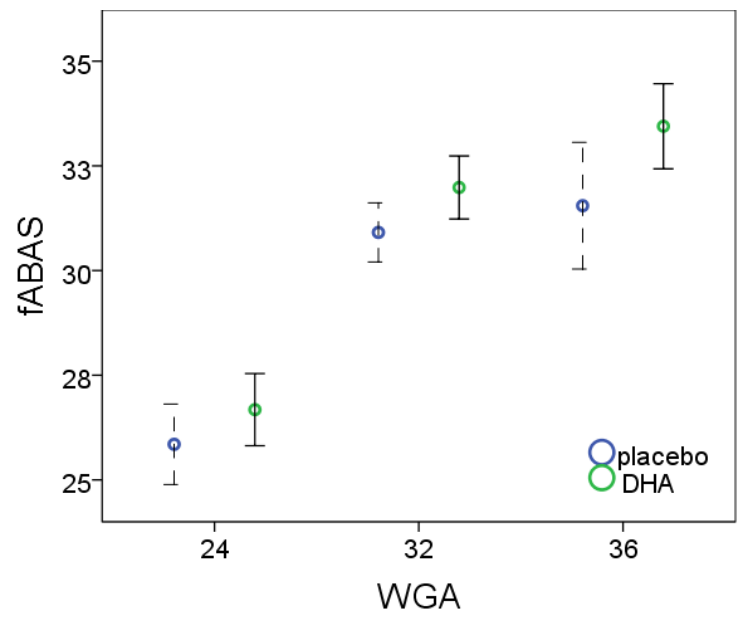

Figure 1: fABAS values, DHA versus placebo group, 24, 32,36 WGA, active sleep $2 \mathrm{~F}$, mean $\pm 95 \% \mathrm{CI}$. 
fABAS was significantly increased in the DHA group at 32 and 36 WGA, but at 24 WGA only by tendency (Figure 1, Table 1). Considering the coefficients in univariate way, pNN5 was increased in the DHA group at 32 and 36 WGA, while ActAmp at 36 WGA only (Table 1). The distribution of the discriminatory strength of the univariate coefficients was different from that of their contribution to the physiological age estimation expressed by the beta values (standardized regression coefficients) in the fABAS model.

Table 1. Standardised regression coefficients beta of the factors within fABAS and their individual t-test results of DHA vs. placebo.

\begin{tabular}{lllll}
\hline & $\begin{array}{c}\text { Age fit } \\
\text { fABAS } \\
\text { beta }\end{array}$ & \multicolumn{3}{c}{$\begin{array}{c}\text { DHA vs. placebo } \\
\text { test results } \\
\text { p values }\end{array}$} \\
\hline fABAS & & 24 WGA & 32 WGA & 36 WGA \\
ActAmp & 0.39 & 0.18 & $\mathbf{0 . 0 4}$ & $\mathbf{0 . 0 4}$ \\
skew & 0.19 & 0.08 & 0.34 & $\mathbf{0 . 0 1}$ \\
pnn5 & 0.16 & 0.80 & $\mathbf{0 . 0 1}$ & $\mathbf{0 . 0 4}$ \\
VLF/LF & 0.10 & 0.1 & 0.69 & 0.93 \\
gMSE3 & 0.27 & 0.48 & 0.18 & 0.62 \\
\hline
\end{tabular}

The values z-transformed with respect to the physiological reference group seems to be an instructive tool for the presentation of developmental deviations such as those associated with maternal DHA supplementation, for example.

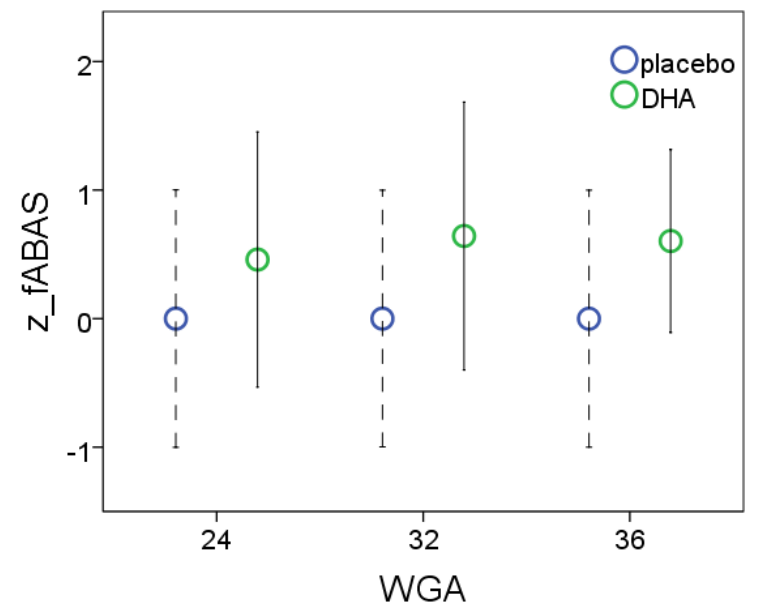

Figure 2: fABAS deviation of DHA from placebo group, expressed by z-score normalized to placebo group in a modified forest plot, 24, 32, 36 WGA, active sleep $2 \mathrm{~F}$, mean $\pm \mathrm{SD}$.

\section{Discussion}

The results show increased fABAS linked to maternal DHA supplementation as we expected. With respect to methodological details, we discuss the consideration of the fetal behavioral states and speculate on interpretation possibilities of the discriminating coefficients of fABAS.

The formation of different activity and behavioral states is part of the fetal development. Starting from about 20 WGA, periods of rest and activity form and become increasingly discernable. Behavioral states, namely quiet and active sleep $(1 \mathrm{~F}, 2 \mathrm{~F})$ and wakefulness $(3 \mathrm{~F}, 4 \mathrm{~F})$ develop and can be discriminated after 32 WGA [10]. Both, the autonomic dynamics across the total behavior and the dynamics within the particular states depend on the maturation age $[11,12]$. Since a recording of $30 \mathrm{~min}$ is only a snapshot that cannot contain all possible states and the fetus stays more than $50 \%$ of time in $2 \mathrm{~F}$, the results of $2 \mathrm{~F}$ seem to be predestinated for routine use. In many subjects we did not find a $1 \mathrm{~F}$ section. In the stateindependent analysis the results were dispersed due to the unknown and possibly changing states. In that regard recordings over $24 \mathrm{~h}$ might be helpful, but they are not practicable for routine use. Also, guidelines of the prenatal care and risk assessment using CTG recordings propose the analysis of active periods. Moreover, they rate the lack of an active section in a 60 min recording as suspicious [9]. Inevitable physiological limitations in the state classification base on their immature manifestation and the biological variability. Neither the subjective consensus decision nor complicated signal analyses can overcome the discrepancy between the idealized 4 states in a stationary non-stress situation and the physiological reality.

Evolution takes place continuously and stepwise. Connections over increasing space and time develop and self-organize complex coordinated behavior. According to neuroanatomical studies sequential periods of ANS development were suggested. At the end of the second trimester the differentiation of the lateral zone of the hypothalamus as well as an increasing myelination of the vagus nerve indicate a predominant development of the parasympathetic branch. From 32 WGA onwards evolving baroreflex sensitivity and respiratory sinus arrhythmia indicate advancing vagal control. Simultaneously, the formation of sympathetic control and the coordination of sympathetically mediated heart rate accelerations and fetal movements indicate the increasing complex integration of sympatho-vagal autonomic control of the developing organism. 
DHA stimulates neuronal growth and cellular communication as well as the increase of cellular energy levels for nerve transmission [6]. Consistently, fABAS was increased at 32 and 36 WGA. Furthermore, at these stages of the pregnancies, DHA supplementation had been sustained considerably longer than at 24 WGA and this may lead to a cumulative promoting effect on the progressively developed sympatho-vagal integration.

All HRV indices used in fABAS address particular aspects that are related to both, the sympatho-vagal development and the universal characteristics of evolution. The consideration of their individual contribution as factors of fABAS may allow interpretations with respect to developmental deviations compared to the physiological aging process in more detail.

The beta values quantify the physiological increase of all these factors as gestation progresses. Compared to the physiological aging, the selective increases of pNN5 and AMP indicate an asynchronous "aging" effect in association with DHA supplementation. Specifically, the increases of pNN5 by 32 WGA and AMP by 36 WGA appear to be consistent with the periods of physiological increase of the vagal and integrative sympatho-vagal activity [12]. Beyond the seemingly robust meaningfulness of the total fABAS, the attempt to interpret the details raises several questions on the statistical modelling and the complex effects of DHA on the materno-fetal system that have to be addressed in further research.

\section{Summary and Conclusions}

fABAS fitted at a larger normal population of the Jena University Hospital data base was able to show an enhancing effect of DHA supplementation on the development of the autonomic control in a randomized, double-blind, placebo-controlled study data set from the University of Kansas Medical Center. This effect is concomitant with the advancing vagal and sympathovagal integrative control in the third trimester.

\section{Acknowledgements}

DH, US, and AS were supported by German Research Foundation: 'Development of a clinic suitable marker of fetal autonomic maturation' (DFG: Ho 1634/15-12, Schn 775/7-1), 'Biomagnetic investigations of fetal autonomic and central nervous maturation and its disturbances due to intrauterine growth restriction and glucocorticoide administration (DFG: Ho 1634/12-2, Schn 775/2-3)'.

KFG was supported by The Eunice Kennedy Shriver National Institute of Child Health and Development (R21 HD059019)

\section{References}

[1] Barker DJ. In utero programming of chronic disease. Clinical science. 1998;95:115-28.

[2] Hoyer D, Tetschke F, Jaekel S, Nowack S, Witte OW, Schleussner E, et al. Fetal functional brain age assessed from universal developmental indices obtained from neuro-vegetative activity patterns. PLoS One. 2013;8(9):e74431.

[3] Nijhuis IJ, ten Hof J, Mulder EJ, Nijhuis JG, Narayan H, Taylor DJ, et al. Fetal heart rate in relation to its variation in normal and growth retarded fetuses. Eur $\mathrm{J}$ Obstet Gynecol Reprod Biol. 2000;89:27-33.

[4] Ferrario M, Signorini MG, Magenes G. Complexity analysis of the fetal heart rate variability: early identification of severe intrauterine growth-restricted fetuses. Med Biol Eng Comput. 2009;47:911-9.

[5] Hoyer D, Schneider U, Kowalski EM, Schmidt A, Witte $\mathrm{OW}$, Schleussner E, et al. Validation of functional fetal autonomic brain age score fABAS in $5 \mathrm{~min}$ short recordings. Physiol Meas. 2015;36:2369-78.

[6] Gustafson KM, Carlson SE, Colombo J, Yeh HW, Shaddy $\mathrm{DJ}, \mathrm{Li} \mathrm{S}$, et al. Effects of docosahexaenoic acid supplementation during pregnancy on fetal heart rate and variability: a randomized clinical trial. Prostaglandins, leukotrienes, and essential fatty acids. 2013;88:331-8.

[7] Gustafson KM, Colombo J, Carlson SE. Docosahexaenoic acid and cognitive function: Is the link mediated by the autonomic nervous system? Prostaglandins, leukotrienes, and essential fatty acids. 2008;79:135-40.

[8] David M, Hirsch M, Karin J, Toledo E, Akselrod S. An estimate of fetal autonomic state by time-frequency analysis of fetal heart rate variability. J Appl Physiol. 2007; 102:1057-64.

[9] FIGO. (Federation Internationale de Gynecologie et d'Obstetrics), Maternity Guidelines, Federal Gazette, Institute for Quality and Efficiency in Health Care, Germany. 2011.

[10] Nijhuis JG, Prechtl HF, Martin CB, Jr., Bots RS. Are there behavioural states in the human fetus? Early Hum Dev. 1982;6:177-95.

[11] Van Leeuwen P, Geue D, Lange S, Hatzmann W, Gronemeyer D. Changes in the frequency power spectrum of fetal heart rate in the course of pregnancy. Prenat Diagn. 2003;23:909-16.

[12] Schneider U, Bode F, Schmidt A, Nowack S, Rudolph A, Doelcker EM, et al. Developmental milestones of the autonomic nervous system revealed via longitudinal monitoring of fetal heart rate variability. Plos One. 2018; 13(7): e0200799.

Address for correspondence.

Prof. Dr. Dirk Hoyer

Biomagnetic Center

Hans Berger Department of Neurology

Jena University Hospital

Am Klinikum 1

D 07747 Jena, Germany

Dirk.Hoyer@med.uni-jena.de 